\title{
Devenir membre d'une communauté de discours : Enquête sur les besoins et les pratiques des docteur-es en sciences
}

Laura M. Hartwell

\section{(2) OpenEdition \\ 1 Journals}

Édition électronique

URL : http://journals.openedition.org/rdlc/1645

DOI : $10.4000 /$ rdlc. 1645

ISSN : 1958-5772

Éditeur

ACEDLE

Référence électronique

Laura M. Hartwell, « Devenir membre d'une communauté de discours : Enquête sur les besoins et les pratiques des docteur-es en sciences $»$, Recherches en didactique des langues et des cultures [En ligne] 10-2 | 2013, mis en ligne le 07 juin 2013, consulté le 21 avril 2019. URL : http:// journals.openedition.org/rdlc/1645; DOI : 10.4000/rdlc. 1645

Ce document a été généré automatiquement le 21 avril 2019

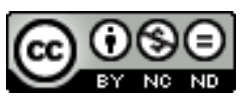

Recherches en didactique des langues et des cultures is licensed under a Creative Commons AttributionNonCommercial-NoDerivatives 4.0 International License 


\title{
Devenir membre d'une communauté de discours : Enquête sur les besoins et les pratiques des docteur-es en sciences
}

\author{
Laura M. Hartwell
}

\section{Introduction}

1 Le Conseil de l'Europe s'appuie sur les universités européennes pour l'apprentissage des langues et le brassage des cultures depuis des décennies. En Europe, nous constatons que les programmes universitaires de l'enseignement des langues pour les spécialistes d'autres disciplines que les langues (LanSAD) sont fortement orientés vers des intérêts académiques et professionnels (Hartwell, 2012). En France, l'officialisation de l'exigence linguistique du mouvement européen pour l'insertion professionnelle et la mobilité fut l'arrêté du 25 avril 2002 stipulant que le diplôme de master « ne peut être délivré qu'après validation de l'aptitude à maîtriser au moins une langue étrangère » (arrêté du 25 avril 2002 relatif au diplôme national de master - NOR : MENS0200982A). Nous verrons que des critères d'évaluation ont été établis par des instances internationales, mais la manière dont un établissement universitaire prend en compte les critères du niveau B2 pour le diplôme de master reste imprécise dans les consignes du ministère. On peut citer l'exemple de l'obligation de valider le niveau B2 en langues pour la titularisation des enseignants. Jusqu'en 2014, les lauréats des concours peuvent produire un diplôme national de master délivré après la validation de l'aptitude à maîtriser une langue vivante étrangère conformément au troisième alinéa de l'article 6 de l'arrêté du 25 avril 2002 relatif au diplôme national de master.

2 Afin de répondre à l'arrêté relatif au diplôme, nous avons consulté les documents de l'Association of Language Testers in Europe (Alte) qui, dans une optique de 
plurilinguisme, propose un ensemble des normes communes pour l'évaluation des compétences langagières. D'autres établissements français s'appuyant sur l'ALTE sont l'Alliance française, le Centre international d'études pédagogiques et la Chambre de commerce et d'industrie de Paris (ALTE, n.d.).

Cette exigence correspond pour l'Université Joseph Fourier (UJF) au niveau B2 du cadre européen de référence (Annexe 1). Depuis trois ans, le corps enseignant de l'établissement évalue le niveau d'environ 700 étudiant-es en deuxième année de master lors des examens, des projets de classe et des oraux devant un jury avec un mode d'évaluation en commun. Trois compétences sont retenues pour un public étudiant poursuivant des études en biologie, chimie, informatique, génie mécanique, maths, physique, pharmacie, sciences de l'univers ou sports et activités physiques :

1. Être capable de faire un exposé clair sur un sujet connu et de répondre à des questions factuelles prévisibles

2. Être capable de parcourir un texte pour retrouver l'information pertinente et d'en saisir l'essentiel

3. Être capable de prendre des notes simples et d'en faire un raisonnable usage pour écrire une dissertation ou faire une révision.

4 Il s'agit de compétences de communication, c'est-à-dire englobant des « connaissances linguistiques et pragmatiques que l'apprenant a intériorisées et qui lui permettent de comprendre et de produire des messages» (Narcy-Combes, 2005). Depuis trois ans, le corps enseignant évalue ces trois compétences de communication à travers des tâches mises en place dans le cadre des études en master scientifique à l'UJF. Le projet d'évaluation est bâti sur l'objectif d'équiper les étudiant-es selon leurs besoins futurs en anglais dans leurs domaines scientifiques, notamment en lecture d'article scientifique, en présentation orale et en prise de notes et non pas en anglais comme langue véhiculaire générale. Ces trois compétences ont été choisies de manière non-empirique, car pour cet établissement, comme pour ailleurs «il n'existe que trop peu d'enquêtes précises sur l'employabilité des étudiants maîtrisant une langue étrangère » dans l'Europe de l'ouest (Thematic Network Project in the Area of Languages, 2008).

5 Dans la tradition en linguistique appliquée de l'analyse des besoins (Flowerdew, 1996; Hyland, 2005 ; Swales, 2004), la présente étude essaye de combler cette lacune dans le secteur des sciences. Nous nous interrogions sur l'impact des certificats en langues type Certificat de Compétences en Langues de L'Enseignement Supérieur (Cles) ou Test of English for International Communication (Toeic) sur l'embauche ou la vie professionnelle des docteur-es. On peut raisonnablement faire l'hypothèse que cette population a besoin de maitriser certains aspects de la langue, mais lesquelles? Quelle est la portée d'une telle maitrise en langue étrangère?

6 Appuyé sur deux enquêtes institutionnelles en 2011 et 2012, cet article va donc tenter de décrire précisément, le rôle joué en France par les certificats en langues dans l'insertion professionnelle et la mobilité des diplômés-es en science ainsi que les besoins langagiers professionnels. Après un portrait du contexte européen et français, on abordera les aspects méthodologiques de deux enquêtes complémentaires. Puis, on verra successivement deux dimensions importantes : le rapport entre certificat et pratique de l'anglais, puis les besoins et les pratiques de trois compétences du point du vue des entreprises et des scientifiques. 


\section{Mouvement européen pour l'insertion professionnelle et la mobilité}

\section{Insertion professionnelle}

7 Pour beaucoup de scientifiques en France comme en Europe, l'anglais est devenu une lingua franca. La maîtrise de l'anglais est une nécessité si l'on veut participer pleinement à des organisations européennes aussi diverses que l'European Academy of Anaesthesiology, l'European Academy of Facial Surgery, l'European Association of Cancer Research et l'European Association of Fish Pathology, qui toutes publient les actes uniquement en anglais (Crystal, 2003: 88). Au sein des entreprises, l'anglais apporte une image "mondiale», bien que les pratiques internes soient variables (Currivand et Truchot, 2009).

8 Mais les études européennes montrent que les scientifiques non anglophones ont parfois des lacunes lorsqu'il faut pratiquer l'anglais. Une étude auprès de chercheurs confirmés à l'Université de Santiago de Compostela montre un multilinguisme chez l'ensemble des chercheurs avec des besoins importants en anglais pour la lecture et la communication orale dans toutes les disciplines et pour la rédaction liée à la recherche dans les sciences expérimentales (Fernández Polo \& Cal Varela, 2009 : 162). De manière comparable, Beyene et al (2009) ont étudié les comportements des locuteurs non natifs basés en Allemagne, aux États-Unis, et en Inde dans une entreprise scientifique allemande pour laquelle la lingue franca est l'anglais. Elles ont constaté trois stratégies pour éviter une surcharge émotionnelle: le retrait des non-natifs lors des discussions, l'exclusion par les germanophones des membres de l'équipe anglophones natifs lors des moments de communication et enfin un va-et-vient entre l'anglais et la langue maternelle.

Les besoins en langue étrangère touchent toutes les compétences. La lecture d'articles en anglais est une tâche récurrente chez les chercheurs en France, comme ailleurs dans le monde (Truchot, 1997). Mais une inquiétude face à l'oral se manifeste aussi parmi les étudiants en France selon plusieurs sondages. Une enquête au Centre de Ressources de Langues de l'Université Louis Pasteur à Strasbourg révèle que l'objectif des étudiant-es se porte «massivement» sur la compréhension de l'oral (Poteaux, 2003: 138-139). À l'Université de Lyon une enquête auprès des étudiant-es en première année révèle que $51,7 \%$ de la population consultée pense que la compréhension de l'oral est la compétence la plus importante à maîtriser, alors que seulement 10 \% estiment la maîtriser (Guichon, 2003). Lavinal et al (2005) montrent que la plus grande difficulté pour les étudiant-es en première année de l'Institut Universitaire de Technologie est « l'écoute » (plus de $40 \%$ ). Enfin, Taillefer (2007) confirme que, selon son sondage, plus de $80 \%$ des diplômés en master en sciences de l'économie à l'Université de Toulouse estiment avoir besoin d'un niveau $\mathrm{B} 2$ ou $\mathrm{C} 1$ dans les quatre compétences et qu'un tiers déclare avoir besoin d'une deuxième langue étrangère.

10 Le besoin de maîtriser une langue étrangère ne se limite pas aux scientifiques. L'Association Pour l'Emploi des Cadres (Apec) a révélé qu'en 2006, $34 \%$ des offres d'emploi sur un échantillon de 122784 offres demandaient au moins une langue étrangère, et ceci à un niveau confirmé pour 81 \% d'entre elles (Taillefer, 2008 : 55). Être démuni dans la langue de son entreprise ou de sa communauté de recherche peut avoir des répercussions étendues, dont une diminution de la mobilité. 


\section{La mobilité}

11 Le Conseil de l'Europe soutient politiquement la mobilité au sein de l'Europe depuis des décennies. En effet, le Joint Study Programme, établi en 1976, fut remplacé par le Programme Erasmus d'étude en Europe en 1987. En 1998, la Déclaration de la Sorbonne souligne le besoin de nouveaux efforts pour réduire les barrières linguistiques et culturelles et pour augmenter la mobilité et la coopération entre les communautés universitaires en Europe. Ces objectifs furent confirmés par la Déclaration de Bologne en 1999 (Räisänen \& Fortanet-Gomez, 2008 : 14-15). Lors de la Recommandation de décembre 2006, le Parlement Européen et le Conseil de l'Europe ont réitéré le rôle clé de l'apprentissage de langues étrangères dans l'amélioration de l'emploi précisant que :

Les aptitudes essentielles à la communication en langues étrangères consistent en la faculté de comprendre des messages oraux, d'amorcer, de poursuivre et de terminer des conversations et de lire, comprendre et rédiger des textes répondant aux besoins de l'individu. Les individus devraient également être capables d'utiliser correctement les techniques de support, et d'apprendre des langues de façon informelle au titre de l'éducation et de la formation tout au long de la vie. Une attitude positive implique une sensibilité à la diversité culturelle, et un intérêt et une curiosité pour les langues et la communication interculturelle (Journal officiel de l'Union européenne, 2006 : 394/15)

La mobilité de la population étudiante française dans le programme Erasmus est en augmentation continuelle, passant de 25945 en 2007-2008 à 31747 en 2010-2011,

En 2010, 1,41 \% de la population étudiante française a étudié dans le cadre Erasmus dans un des 32 pays participants, plaçant la France en $13^{\mathrm{e}}$ place entre l'Islande $(1,46 \%)$ et l'Irlande (1,29\%). Ceci reflète l'augmentation de la population étudiante, car en 2000 , $1,6 \%$ de la population étudiante française a étudié dans un autre des 27 pays de l'Europe, la plaçant en $19^{\mathrm{e}}$ place avec les Pays Bas. Puis, en 2006, 2,2 \% des Français-es ont étudié dans un pays de l'Europe, c'est-à-dire en $20^{\mathrm{e}}$ place avec la Lettonie. Le contexte intérieur du pays a un effet direct sur la mobilité, par exemple, les universités de Luxembourg et de Liechtenstein offrent moins de choix disciplinaires, mais ont des taux de mobilité importants ( $8,2 \%$ et $4,8 \%$ respectivement en 2010$)$. En contraste, La Croatie $(0,39 \%)$ et la Romanie $(0.46 \%)$ a peu participé, mais leurs chiffres de participation sont en net augmentation comparé aux années précédentes. En 2010, seul Malte n'a pas participé au programme. Les étudiant-es du Royaume-Uni sont systématiquement ceux qui voyagent le moins (de $0,6 \%$ à $0,5 \%$ entre 2000 et 2010 ), cela peut s'expliquer par leur politique nationale qui promeut peu l'apprentissage de langues étrangères et la pléthore d'établissements universitaires dans le pays (Eurostat, 2009 : 210-211 ; Lifelong Learning Programme, 2012 : 15-23). En l'absence de mobilité étudiante, l'apprentissage des langues dépend davantage des cours.

\section{Méthodologie}

Dans ce cadre, le Service des Langues en conjonction avec l'Observatoire des formations et du suivi étudiant de l'établissement a lancé une enquête auprès des responsables de ressources humaines dans ce secteur et les étudiant-es ayant obtenu leur doctorat en 2008, l'objectif étant de cibler la corrélation entre la formation en langues et les besoins professionnels. La récolte des données s'est faite à travers deux enquêtes écrites, l'une dans le cadre de l'enquête annuelle auprès des docteurs diplômés trois ans plus tôt 
(Annexe 2) et l'autre auprès des personnes liées aux ressources humaines au sein des entreprises qui recrutent les personnes diplômées de l'UJF (Annexe 3).

Les questionnaires ont été conçus avec l'aide d'Évelyne Janeau, Directrice de l'OFE. Un ensemble de questions à réponse oui/non ou à choix multiples a été conçus afin d'encourager les participants à terminer et à soumettre l'enquête. Les quatre compétences «classiques » liées à la compréhension et l'expression de l'écrit et de l'oral ont été complétées par les trois compétences privilégiées par l'établissement : la lecture d'article scientifique, la présentation orale et la prise de notes. Pour les Doc +3 , l'objectif était de connaître la fréquence d'utilisation des différentes compétences en langues étrangères et d'explorer le niveau d'aisance parmi ces sept compétences (Annexe 2). En direction des RRH, le lien entre niveau en langues, certification, embauche et réussite professionnelle a été privilégié (Annexe 3). Une dernière question à réponse ouverte n'est pas traitée, car ayant reçu une seule réponse. Des extraits des questions à réponse ouverte et des analyses statistiques aident à mieux comprendre la portée des données quantitatives.

16 Les résultats des deux enquêtes ont été traités par l'auteure. Je m'appuie aussi sur les données du rapport interne : « Docteurs des écoles doctorales de l' UJF 2004, 2005, 2006, 2007, 20083 ans après " (Janeau, 2012). Bien que les enquêtes annuelles de l'établissement ne posent aucune question concernant la maitrise de langues étrangères, les participants évoquent cette notion à plusieurs reprises. De plus, le rapport offre des informations supplémentaires concernant le contexte global des Doc+3.

\section{Participants}

\section{Les diplômé-es}

L'Observatoire des formations et du suivi étudiant (OFE) a envoyé la première enquête en annexe à son enquête annuelle. Les résultats collectés par l'OFE représentent une population comportant 71 docteur-es diplômé-es en 2008 par les écoles doctorales de l'UJF (désormais Doc+3). Le taux de réponse était de $24,3 \%$, mais reflète, selon l'OFE l'ensemble de 325 docteurs ayant soutenu leur diplôme en 2008 et peut donc apporter des réponses significatives (Janeau, courriel personnel, 2012). Pour l'ensemble des docteurs diplômés dans les domaines scientifiques, on trouve $36,3 \%$ de femmes et $73,7 \%$ d'hommes, $68,7 \%$ de personnes de nationalité française et $31,3 \%$ d'autres nationalités, enfin $78 \%$ des participants avaient moins de 30 ans lors de la soutenance de thèse. En moyenne, plus de la moitié $(56,3 \%)$ des sujets étaient en Contrat à Durée Indéterminée (CDI) ou fonctionnaire, $32,8 \%$ en Post Doctoral et 7,9\% en Contrat à Durée Déterminée (CDD). La précarité en début de carrière touche donc plus d'un tiers de cette population, ce qui reflète l'augmentation de la précarité dans l'enseignement supérieur en France (Collectif PAPERA, 2012). En dehors de la France, les pays d'accueil les plus populaires pour un contrat de Post Doctoral sont les États-Unis (6,6 \%) et le Canada (2,7 \%).

\section{Les professionnels}

La population de la deuxième partie de l'étude est composée des personnes ayant une grande expérience dans le monde professionnel des scientifiques. Philippe Chinkirch, membre de Valoridoc, une association destinée à l'insertion professionnelle des diplômés de l'UJF a été mandaté par l'OFE à envoyer l'enquête auprès d'une sélection des 
Responsables de Ressources Humaines en entreprise (désormais RRH) en mai 2011. Sept personnes ont répondu dans un délai de quatre mois, dont une responsable d'Administration et de Finance dans une Petite et Moyenne Entreprise (PME), une consultante dans un cabinet, deux responsables des ressources humaines, dont une dans un groupe international, et trois responsables de PME. Les données m'ont été transmises sans analyse ou commentaire. Les professionnels n'ont pas pris le temps de répondre aux questions à réponses ouvertes.

\section{Résultats : l'obtention d'un certificat et la fréquence de pratique de l'anglais}

\section{L'obtention d'un certificat en langues}

L'ensemble des RRH a indiqué qu'un «bon niveau d'anglais" a un effet positif sur l'embauche et la performance professionnelle. Selon cette population, l'importance de l'obtention d'un certificat en langue est moins évidente. Un Toeic (avec un score de 750 ou plus) est considéré comme un point positif pour cinq RRH et un certificat International English Language Testing System (IELTS) 5+ comme un avantage par quatre RRH. Enfin, pour trois RRH, un Cles2 ou la validation du niveau B2-Alte études par (nom supprimé afin de préserver l'intégrité du processus de sélection) sont aussi considérés comme un élément positif pour l'embauche. On note néanmoins un manque de connaissances chez les grandes entreprises scientifiques concernant le cadre européen commun de références en langues élaboré par la commission européenne, et adopté par l'éducation nationale française depuis 2005. De ce fait, le certificat de compétences en langues de l'enseignement supérieur (Cles) et le système de validation interne de compétences en anglais au niveau « utilisateur indépendant » (B2) mis en œuvre par (nom supprimé afin de préserver l'intégrité du processus de sélection) ne sont pas reconnus. Deux des RRH ont aussi noté l'effet positif d'un "autre certificat ou langue » sans préciser lesquels. En revanche, aucun RRH ne pense que le temps de préparation à ces certificats ait un effet positif sur la performance professionnelle et un RRH pense même que cela a un effet négatif, qu'il s'agisse de la préparation au Toeic, qui bénéficie d'une certaine renommée, ou à d'autres certificats qui sont moins connus. C'est-à-dire que, selon ces professionnels des entreprises privées, l'entrainement nécessaire à l'obtention de ces certificats ne se traduit pas par une meilleure performance professionnelle en langues étrangères. On peut supposer que l'obtention d'un certificat reflète favorablement la volonté du candidat-e, mais que la langue générale présente dans les certificats des langues ne correspond pas aux exigences des milieux scientifiques.

\section{Rapport entre l'obtention d'un certificat en langues et la pratique de la langue}

20 Nous avons alors tenté de voir si l'obtention d'un certificat en langues est corrélée avec une pratique accrue de l'anglais au niveau professionnel chez les Doc +3 . Parmi la population consultée, personne n'a déclaré avoir obtenu un certificat Cles ou Ielts. L'affectation d'un barème aux réponses de la question 2 (fréquence d'utilisation de sept compétences en anglais) combinée avec la possession d'un certificat spécifique en langues permet de construire quatre groupes de fréquence d'utilisation différents des 
compétences en anglais : de la fréquence la plus faible à la fréquence la plus élevée. Le barème est construit selon la cotation : rarement $=0,25$; plusieurs fois par an 0,50 ; plusieurs fois par mois $=0,75$; tous les jours $=1,00$. Le Tableau 1 montre que ce public de personnes possédant un certificat est régulièrement distribué entre " très faible à faible " $(25,5 \%)$ à une utilisation «forte à très forte » $(16,5 \%)$ avec une concentration dans la catégorie d'utilisation « moyenne à forte » (39\%). La possession d'un certificat Toeic n'est corrélée ni avec une plus forte utilisation de l'anglais, ni avec un poste de professeur assistant dans le supérieur à l'étranger au moment de la réponse à l'enquête (Tableau 1).

Tableau 1 - Fréquence d'utilisation de l'anglais et la possession d'un certificat ou pratique d'une autre langue

\begin{tabular}{|c|c|c|c|c|c|}
\hline $\begin{array}{l}\text { Situation } \\
\text { Utilisation d'anglais }\end{array}$ & Toeic & Toefl & $\begin{array}{l}\text { Autre : } \\
\text { non } \\
\text { précisé }\end{array}$ & $\begin{array}{lr}\text { Total des } \\
\text { certificats ou } \\
\text { autre langue }\end{array}$ & $\begin{array}{l}\text { Professeur assistant dans } \\
\text { l'enseignement supérieur à } \\
\text { l'étranger }\end{array}$ \\
\hline $\begin{array}{l}\text { Très faible à faible } \\
17 \quad \text { personnes } \\
(25,5 \%)\end{array}$ & \begin{tabular}{|l}
4 \\
$23,5 \%$
\end{tabular} & $\begin{array}{l}4 \\
23,5 \%\end{array}$ & 0 & $\begin{array}{l}8 \\
27 \%\end{array}$ & 1 \\
\hline $\begin{array}{l}\text { Faible à moyenne } \\
13 \quad \text { personnes } \\
(19,5 \%)\end{array}$ & 0 & $\begin{array}{l}3 \\
23,1 \%\end{array}$ & 0 & $\begin{array}{l}3 \\
23,1 \%\end{array}$ & 2 \\
\hline $\begin{array}{l}\text { Moyenne à forte } \\
26 \text { personnes (39\%) }\end{array}$ & $\begin{array}{l}5 \\
19,2 \%\end{array}$ & $\begin{array}{l}2 \\
7,7 \%\end{array}$ & $\begin{array}{l}1 \\
3,8 \%\end{array}$ & $\begin{array}{l}8 \\
30,8 \%\end{array}$ & 1 \\
\hline $\begin{array}{l}\text { Forte à très forte } \\
11 \quad \text { personnes } \\
(16,5 \%)\end{array}$ & $\begin{array}{l}2 \\
18,2 \%\end{array}$ & 0 & $\begin{array}{l}3 \\
27,3 \%\end{array}$ & $\begin{array}{l}5 \\
45,5 \%\end{array}$ & 0 \\
\hline Total & 11 & 9 & 4 & $24^{*}$ & 4 \\
\hline
\end{tabular}

* Sur les 79 participants, 24 ont obtenu un certificat en langues.

21 Afin d'analyser les relations entre l'obtention d'un certificat et la fréquence de l'utilisation de l'anglais, nous avons réalisé un test du chi-carré. La fréquence d'utilisation de l'anglais en fonction de leur possession ou non d'un certificat ne s'écarte pas de manière significative de la distribution théorique: 03, $p>0,50$. Autrement dit, les variables «certification» et "utilisation forte» ne semblent pas être reliées. En appliquant la correction de Yates, la valeur du chi-carré reste non significative $\left(^{2}=0,148\right.$, $p>0,50$ ). Le calcul de Cramer confirme ce résultat. Avec une valeur de 0,063, il signale une indépendance quasi totale entre les deux variables. En résumé, il n'y a pas de différence significative entre l'obtention d'un certificat (Toeic, Test Of English as a Foreign Language (Toefl), ou autre certificat en langues) et une pratique accrue de l'anglais au niveau professionnel pour ce public. C'est-à-dire que l'obtention d'un certificat en langue est déconnectée du fait d'intégrer une communauté scientifique, car l'objectif d'obtention d'un certificat n'est pas lié à une véritable pratique langagière sur le terrain. 
Le Toeic est présenté par Educational Testing Service (ETS) comme étant un certificat à objectif professionnel (http://www.ets.org/Toeic/institutions). Nous venons de voir qu'il n'y a pas de corrélation entre l'obtention d'un certificat et la fréquence de pratique de l'anglais. Maintenant, nous examinons la relation entre l'obtention d'un certificat et l'aisance de la pratique de l'anglais à partir d'un t-test. Nous avons comptabilisé les déclarations des difficultés dans les sept compétences visées par la question $3 \mathrm{du}$ questionnaire (lire, écouter, écrire, parler, lire des articles scientifiques, présenter oralement et prendre des notes) (Annexe 2). Un t-test basé sur ces sept critères n'indique aucune différence significative $(p=0,718)$ des difficultés en anglais pour un sousensemble de 67 Doc+3 venant de 5 écoles doctorales à (nom supprimé afin de préserver l'intégrité du processus de sélection) $(M=5,11 ; E T=2,02)$ et les 12 personnes de ce groupe qui possèdent un certificat Toeic $(\mathrm{M}=5,33 ; \mathrm{ET}=1,83)$. C'est-à-dire, il n'y a pas de différence significative entre une plus grande facilité en anglais chez les personnes possédant un Toeic et le reste de cette population. La préparation, voire le bachotage, au Toeic ne semble pas améliorer l'aisance en anglais des candidat-es.

L'obtention d'un certificat n'est pas forcement nécessaire, car il n'est ni en rapport avec une pratique plus fréquente de la langue, ni avec une plus grande aisance lors de la pratique. Il reste à savoir comment cette pratique de l'anglais se manifeste dans le discours des sujets ayant répondu aux questionnaires qu'il y ait obtention d'un certificat ou non. Pour cela, les résultats des questionnaires seront présentés par compétence : la compréhension de l'écrit, l'expression orale et la prise de notes. Bien que cette séparation en catégories par compétence soit artificielle, cette approche aide à mieux discerner les caractéristiques des besoins.

\section{Résultats : besoins et pratiques de trois compétences}

La vaste majorité des participants affirme que la lecture de textes en anglais est un acte quasi quotidien (Tableau 2). La lecture de textes en anglais général prend une place plus importante que la lecture d'articles scientifiques. Ceci est exprimé par les Doc+3, dont 62 (78,5\%) disent lire tous les jours et $52(65,8 \%)$ qui disent lire des articles scientifiques tous les jours. Similairement, les RRH trouvent unanimement que "lire " est d'une importance élevée sur le plan professionnel, mais avec des réponses beaucoup plus mitigées en ce qui concerne la lecture d'articles scientifiques. Les réponses des RRH indiquent que la lecture de textes en anglais général se fait au moins plusieurs fois par mois. La lecture d'articles scientifiques est moins fréquente, ce qui peut être davantage une différence entre les besoins des personnes diplômées en master comparé aux Doc+3, comme explique un-e Doc +3 , la lecture est «Utile pour la recherche, mais pas pour l'obtention d'un travail hors de la recherche » (Annexe 4).

Tableau 2 - Fréquence et type de lecture

\begin{tabular}{|l|l|l|l|l|l|l|}
\hline Participants & $\begin{array}{l}\text { Type } \\
\text { lecture }\end{array}$ & $\begin{array}{l}\text { Tous } \\
\text { les } \\
\text { jours }\end{array}$ & $\begin{array}{l}\text { Plusieurs fois } \\
\text { par mois }\end{array}$ & $\begin{array}{l}\text { Plusieurs } \\
\text { fois par an }\end{array}$ & Rarement & Total \\
\hline Docteurs +3 & Général & 62 & 11 & 4 & 2 & 79 \\
\hline
\end{tabular}




\begin{tabular}{|c|c|c|c|c|c|c|}
\hline & $\begin{array}{l}\text { Articles } \\
\text { scientifiques }\end{array}$ & 52 & 13 & 8 & 6 & 79 \\
\hline $\begin{array}{l}\text { Responsables en } \\
\text { Ressources Humaines } \\
\text { (RRH) }\end{array}$ & Général & 6 & 1 & & & 7 \\
\hline & $\begin{array}{l}\text { Articles } \\
\text { scientifiques }\end{array}$ & & $\begin{array}{l}4 \\
1 \text { technique }\end{array}$ & 1 & 2 & 7 \\
\hline
\end{tabular}

Bien qu'omniprésente, la lecture présente parfois des difficultés (Tableau 3). Au niveau des diplômé-es en master, les RRH soulignent qu'un niveau insuffisant en lecture en anglais est un facteur négatif et déterminant lors de l'embauche. Les Doc +3 ont exprimé des réponses parfois contradictoires en ce qui concerne cette compétence. Si la vaste majorité estime ne pas avoir de difficulté en lecture, presque la moitié $(n=39,49,4 \%)$ reconnaissent le besoin de progresser en lecture d'anglais général, mais aussi en lecture d'articles scientifiques ( $n=35 ; 44,3 \%$ ). Un-e Doc+3 décrit la nécessité de mieux maîtriser la compréhension de l'écrit : « On en a toujours besoin. Autant en faire pas mal pour vite arriver à ne plus avoir besoin de lire une publication avec un dictionnaire à côté ! »

Tableau 3 - Difficultés et besoin de progresser en lecture

\begin{tabular}{|l|l|l|l|l|l|l|}
\hline & & Difficultés & $\begin{array}{l}\text { Parfois des } \\
\text { difficultés }\end{array}$ & $\begin{array}{l}\text { Pas de } \\
\text { difficultés }\end{array}$ & $\begin{array}{l}\text { Besoin de } \\
\text { progresser }\end{array}$ & Total \\
\hline $\begin{array}{l}\text { Docteurs } \\
+3\end{array}$ & Général & - & 8 & 71 & 39 & $79^{*}$ \\
\hline & $\begin{array}{l}\text { Articles } \\
\text { scientifiques }\end{array}$ & - & 6 & 73 & 35 & $79^{*}$ \\
\hline
\end{tabular}

* Ce tableau regroupe deux questions, celle concernant la difficulté et celle concernant le besoin de progresser. II peut donc avoir plus de 79 réponses par ligne.

\section{Expression orale}

L'expression orale serait un peu moins souvent pratiquée que la lecture, mais présenterait davantage de difficulté. Un docteur explique «J'étais suffisamment préparé pour lire l'anglais scientifique. Tenir une discussion courte était plus dur » ou encore «Beaucoup de gens oublient qu'il est aussi important de savoir bien communiquer en anglais. [La présentation orale] est un bon exercice surtout si le sujet de communication est en rapport avec les cours ou les travaux de recherche. » Cinq sur sept RRH pensent que « parler » est d'une importance élevée au niveau professionnel et six sur sept pensent que des lacunes dans cette compétence peuvent être un frein à l'embauche. La place de la présentation orale est moins établie, car seuls deux RRH trouvent que cette compétence est d'une importance élevée ou qu'elle est un frein à l'embauche. 
En ce qui concerne les besoins en expression orale, les constats des Doc+3 sont similaires aux conceptions des besoins notées par les RRH (Tableau 4). «Parler» se fait tous les jours pour $39,2 \%(n=31)$ des Doc +3 et plusieurs fois par mois pour $26,6 \%(n=21)$ des participants. Ce besoin est reflété dans les réponses des RRH, pour lequel quatre sur sept estiment que l'anglais oral se pratique tous les jours et deux autres pour lesquels l'anglais oral se pratique plusieurs fois par mois.

Pour une petite moitié des Doc $+3(n=34 ; 43 \%)$, la nécessité de présenter oralement arrive plusieurs fois par an, mais pour un tiers $(n=23 ; 29,1 \%)$, c'est un acte rare. Bien que les RRH évoquent les besoins des diplômés en master, trois sur sept jugent que le besoin de présenter oralement arrive plusieurs fois par mois.

TABLEAU 4 - Fréquence et type d'expression orale

\begin{tabular}{|c|c|c|c|c|c|c|}
\hline & $\begin{array}{ll}\text { Type de } \\
\text { lecture }\end{array}$ & $\begin{array}{l}\text { Tous les } \\
\text { jours }\end{array}$ & $\begin{array}{l}\text { Plusieurs fois } \\
\text { par mois }\end{array}$ & $\begin{array}{l}\text { Plusieurs } \\
\text { fois par an }\end{array}$ & Rarement & Total \\
\hline \multirow[t]{2}{*}{ Docteurs +3} & Parler & 31 & 21 & 17 & 10 & 79 \\
\hline & Présenter & 9 & 13 & 34 & 23 & 79 \\
\hline \multirow[t]{2}{*}{$\begin{array}{l}\text { Responsables en } \\
\text { Ressources Humaines } \\
\text { (RRH) }\end{array}$} & Parler & 4 & 2 & 1 & - & 7 \\
\hline & Présenter & 1 & 3 & 2 & 1 & 7 \\
\hline
\end{tabular}

Une petite moitié des Doc+3 éprouve des difficultés $(n=15 ; 19 \%)$ ou parfois des difficultés $(n=21 ; 26,6 \%)$ à parler en anglais. En contraste, presque deux tiers $(n=56 ; 71 \%)$ constatent un besoin de progresser en techniques de la présentation orale en anglais (Tableau 5). L'impression d'avoir besoin de progresser est donc beaucoup plus forte qu'en lecture. Un-e Doc+3 exprime des objectifs ambitieux en ce qui concerne un aspect affectif (l'assurance) nécessaire lors des présentations orales : «Très important. Seule la pratique permet d'acquérir une assurance parfaite ». 
Tableau 5 - Difficultés et besoin de progresser à l'expression orale

\begin{tabular}{|c|c|c|c|c|c|c|}
\hline & & Difficultés & $\begin{array}{l}\text { Parfois des } \\
\text { difficultés }\end{array}$ & $\begin{array}{l}\text { Pas de } \\
\text { difficultés }\end{array}$ & $\begin{array}{l}\text { Besoin de } \\
\text { progresser }\end{array}$ & $\begin{array}{l}\text { Total de } \\
\text { répondantes }\end{array}$ \\
\hline \multirow[t]{2}{*}{$\begin{array}{l}\text { Docteurs } \\
+3\end{array}$} & Parler & 15 & 21 & 43 & 56 & $79^{*}$ \\
\hline & $\begin{array}{l}\text { Présenter } \\
\text { oralement }\end{array}$ & 11 & 22 & 46 & 58 & $79^{*}$ \\
\hline
\end{tabular}

* Ce tableau regroupe deux questions, celle concernant la difficulté et celle concernant le besoin de progresser. II peut donc avoir plus de 79 réponses par ligne.

De plus, cette compétence est fortement liée avec des capacités de mobilité et donc de recrutement, comme exprimé par un-e Doc+3 lors de l'enquête de l'OFE (Janeau, 2012) :

Ce n'est pas le cas pour moi, mais j'ai connu beaucoup de thésards qui ne partent pas en congrès internationaux. Or c'est dans ces congrès que les réseaux se construisent pour la future insertion professionnelle (Janeau, 2012 : 12).

\section{Prise de notes}

31 La prise de notes à partir d'une source orale est une compétence complexe, car elle lie simultanément les besoins en compréhensions de l'oral et en production écrite, alors que l'attention est souvent attirée par les supports visuels (Hartwell, 2010 ; Hartwell, 2009). Le Cadre européen commun de référence pour les langues précise qu'une personne de niveau "usager indépendant» (B2) peut comprendre une présentation orale telle qu'une conférence si celle-ci est clairement structurée et si le sujet est familier. La personne peut aussi prendre des notes sur des points qui lui semblent importants, bien qu'elle ait tendance à se concentrer sur les mots et donc puisse ne pas comprendre certaines données (Council of Europe, 2001: 96). Le fait de relever les points pertinents lors de l'écoute implique que l'individu comprend et trie les informations et les concepts et ne se contente pas de les transcrire.

Certains commentaires des Doc +3 montrent que les aspects multiples de la tâche de la prise de notes dans sa globalité sont mal cernés :

La prise de notes est par nature permissive en ce qui concerne les fautes de langue. Je ne pense pas qu'il y ait un effort à faire dans cette direction. Le problème est plus la compréhension de la source orale que la reproduction sous forme de notes.

Cette description souligne l'importance de la compréhension, mais sous-estime les demandes cognitives de la prise de notes. Les problèmes liés à l'écrit, selon cette réponse, seraient limités à une question de grammaire ou d'orthographe et non, par exemple, aux choix liés aux informations à noter ou la manière des les noter afin de pouvoir les exploiter par la suite. Une autre réponse exprime la notion de sélection, une notion essentielle, car la parole est bien plus rapide que l'écrit : 
La prise de notes, on la fait comme on veut. Le plus important c'est de comprendre ce que disent les gens et de prendre les notes qui vous intéressent.

Évaluées de manière séparée, la compréhension de l'oral (écouter) et l'expression écrite sont décrites par plus que la moitié des participants comme étant des actes quotidiens. Chez les Doc+3, 54,4 \% $(n=43)$ écoutent l'anglais au travail tous les jours et 53,2 \% $(n=42)$ l'écrivent tous les jours. Les RRH mettent davantage l'accent sur l'écrit, puisque six sur sept ont répondu qu'il s'agit d'un acte quotidien. C'est considérable. Le sondage n'a pas précisé le type d'écriture demandée, mais l'on peut penser à la rédaction de courriels. Les réponses concernant la prise de notes comme compétence spécifique sont plus mitigées. Un tiers $(n=28 ; 35,4 \%)$ des Doc+3 déclare ne prendre des notes que "rarement », ce qui est aussi reflété par le peu de réponses chez les RRH (Tableau 6).

TABLEAU 6 - Fréquence des compétences liées à la prise de notes

\begin{tabular}{|c|c|c|c|c|c|c|}
\hline Participants & $\begin{array}{l}\text { Type de } \\
\text { lecture }\end{array}$ & $\begin{array}{l}\text { Tous les } \\
\text { jours }\end{array}$ & $\begin{array}{l}\text { Plusieurs fois } \\
\text { par mois }\end{array}$ & $\begin{array}{l}\text { Plusieurs fois } \\
\text { par an }\end{array}$ & Rarement & Total \\
\hline & Écouter & 43 & 24 & 9 & 3 & 79 \\
\hline \multirow[t]{3}{*}{ Docteurs +3} & Écrire & 42 & 18 & 14 & 5 & 79 \\
\hline & $\begin{array}{l}\text { Prise de } \\
\text { notes }\end{array}$ & 20 & 15 & 16 & 28 & 79 \\
\hline & Écouter & 4 & 2 & 1 & - & 7 \\
\hline $\begin{array}{lr}\text { Responsables } & \text { en } \\
\text { Ressources } & \text { Humaines } \\
\text { (RRH) } & \end{array}$ & Écrire & 6 & 1 & - & 1 & 7 \\
\hline & $\begin{array}{l}\text { Prise de } \\
\text { notes }\end{array}$ & - & - & 1 & 1 & 7 \\
\hline
\end{tabular}

Comme pour la présentation orale, les Doc+3 déclarent généralement ne pas avoir de difficultés à l'écrit ou à l'écoute, mais souhaitent progresser dans ces deux domaines (Tableau 7). Alors que seulement 33 Doc+3 (42\%) disent avoir ou avoir parfois des difficultés, 48 (61\%) disent avoir besoin de progresser en compréhension de l'oral. De manière comparable, $27(34,2 \%)$ personnes de cette population déclarent avoir ou avoir parfois des difficultés à l'écrit, mais $49(62 \%)$ disent avoir besoin d'y progresser. On peut se demander si cette incohérence entre "ne pas avoir des difficultés » et "besoin de progresser » est, en fait, un reflet du phénomène d'évitement des difficultés constaté par Beyene et al (2009). C'est-à-dire que l'individu éviterait une situation de communication 
en langue étrangère afin de ne pas se trouver en difficulté. Dans ce cas, la personne n'a pas de difficulté, mais ne serait pas capable de maitriser suffisamment la langue face à un tel contexte.

Tableau 7 - Fréquence et type de compétence liée à la prise de notes

\begin{tabular}{|l|l|l|l|l|l|l|}
\hline & & Difficultés & $\begin{array}{l}\text { Parfois des } \\
\text { difficultés }\end{array}$ & $\begin{array}{l}\text { Pas de } \\
\text { difficultés }\end{array}$ & $\begin{array}{l}\text { Besoin } \\
\text { progresser }\end{array}$ & Total \\
\hline $\begin{array}{l}\text { Docteurs } \\
+3\end{array}$ & Écouter & 6 & 27 & 46 & 48 & $79^{*}$ \\
\hline & Écrire & 6 & 21 & 52 & 49 & $79^{*}$ \\
\hline & $\begin{array}{l}\text { Prise de } \\
\text { notes }\end{array}$ & 8 & 8 & 63 & 31 & $79^{*}$ \\
\hline
\end{tabular}

* Ce tableau regroupe deux questions, celle concernant la difficulté et celle concernant le besoin de progresser. II peut donc avoir plus de 79 réponses par ligne.

Les réponses concernant la prise de notes semblent aussi être parfois contradictoires. La compréhension de l'oral est à la base de la prise de notes. Or 33 Doc+3 (42\%) déclarent avoir ou avoir parfois des difficultés à l'écoute, mais seulement 16 (20,3\%) disent avoir ou avoir parfois des difficultés à la prise de notes. Cette contradiction peut être interprétée de plusieurs façons. Nous avons vu dans le Tableau 6 que la prise de notes est moins fréquente que l'écoute ou l'écriture seule, pour $28 \mathrm{Doc}+3$ la prise de notes se fait rarement. La relative absence de pratique peut se traduire par une absence de difficultés, bien que le besoin de progresser existe. Aussi, lors des conférences, on peut s'appuyer sur les supports visuels afin de contourner des lacunes en compréhension de l'oral. L'inconvénient de cette stratégie est que les supports visuels offrent souvent des détails sans les commentaires nécessaires à la compréhension globale, détails qui sont, quant à eux, donnés par voie orale. La prise de notes marie compréhension de l'oral et écriture, il se peut que les participants la perçoivent à tort, comme étant une compétence à part et moins essentielle

\section{Conclusion}

On aura donc constaté, à travers l'analyse de ces résultats, qu'une bonne maîtrise de l'anglais, voire d'autres langues, est importante dans l'insertion professionnelle des scientifiques. Ceux-ci ont besoin de l'ensemble des compétences : notamment en lecture qui reste plus facilement acquise, en expression orale qui présente davantage de difficultés, bien que moins fréquemment pratiquée et enfin en prise de notes pour laquelle les résultats sont moins homogènes. Au-delà des différences entre les compétences, ces réponses révèlent l'importance de maîtriser l'anglais lors des colloques ou autres expériences internationales, car celles-ci joueraient un rôle essentiel dans le parcours professionnel. En d'autres termes, l'acquisition de compétences en anglais est une étape permettant à l'individu d'intégrer une communauté, une étape essentielle lors de la recherche d'un poste. 

politique institutionnelle en langues étrangères. On voit confirmation de l'adage que tout ce que l'on peut ajouter à son curriculum vitae pour sortir sa candidature du lot commun peut être un point positif. Les données de la présente étude suggèrent que ce n'est pas un certain certificat en langues, mais le fait de maîtriser l'anglais, voire une autre langue étrangère, qui a un effet positif sur l'embauche. On peut également estimer, bien que cela n'ait pas été exprimé par les répondant-es, que les compétences demandées dans le cadre $\mathrm{du}$ Toeic sont éloignées des pratiques langagières sur le terrain. En contraste, les certificats Ielts et Cles qui ciblent les quatre compétences traditionnelles (lire, écrire, écouter, parler) sont moins connus par les employeurs et moins demandés par cette population. Il semble donc y avoir un décalage national entre l'offre de la certification en langues et les besoins professionnels. Au final, le conseil qui peut être donné à d'autres enseignant-es désireux de répondre aux besoins des futurs professionnel-les du public LANSAD est avant tout de vérifier si un besoin en certification existe véritablement. Dans le cas contraire, une étude des besoins des compétences langagières du domaine professionnel s'impose, car elles peuvent être un facteur positif d'intégration.

\section{BIBLIOGRAPHIE}

Association of Language Testers in Europe (Alte). ALTE language for specific purposes (formerly WOLE). Consulté en septembre 2010 : http://www.alte.org/projects/langsp.php.

Bachman, L. F. (2005). "Building and supporting a case for test use". Language Assessment Quarterly, vol. 1, n 1. pp. 1-34.

Bachman, L. F. \& Palmer, A. S. (1996). Language testing in practice. Oxford: Oxford University Press.

Bayene, T. P., Hinds, P. J. \& Durnell Cramton, C. (2009). "Walking through Jelly: Language Proficiency, Emotions, and Disrupted Collaboration in Global Work". Harvard Business School Working Paper. Consulté en avril 2012: http://www.hbs.edu/research/pdf/09-138.pdf.

Collectif pour l'abolition de la précarité dans l'enseignement supérieur, la recherche et ailleurs. Appel des $50000: 2012$ quel changement pour les 50000 précaires de l'ESR ? Consulté en décembre 2012 : http://www.collectif-papera.org/spip.php?article1422.

Council of Europe. (2001). Common European framework of reference for languages: Learning, teaching, assessment. Structured overview of all CEFR scales. Consulté en septembre 2010: http:// www.coe.int/T/DG4/Portfolio/?L=E\&M=/documents_intro/Data_bank_descriptors.html.

Crystal, D. (2003). English as a global language. Cambridge: Cambridge University Press.

Currivand, T. \& Truchot, C. (2009). "Du traitement des langues aux politiques linguistiques dans l'entreprise". Paris : Ecole du Management. Consulté en décembre 2012 : http://ecole.org/fr/ seances/SEM494

Douglas, D. (2000). Assessing languages for specific purposes. Cambridge: Cambridge University Press. Educational Testing Service (ETS). "TOEIC". Consulté en avril 2012 : http://www.ets.org/toeic. 
Lifelong Learning Programme (2012). Erasmus Programme 2010-2011: A Statistical Overview. (Ed.) Directorate-General for Education and Culture. Bruxelles: Commission Européenne. Consulté en février 2013 : http://ec.europa.eu/education/erasmus/doc/stat/1011/report.pdf Fernández Polo, F. J. \& Cal Varela M. (2009). "English for research purposes at the University of Santiago de Compostela: a survey". Journal of English for Academic Purposes, n 8. pp. 152-164.

Flowerdew, J. \& Miller, L. 1996. Lectures in a Second Language: Notes toward a Cultural Grammar. English for Specific Purposes, vol. 15, n² 2, p. 121-140.

Fulcher, G. (2009). "Test use and political philosophy". Annual Review of Applied Linguistics, n² 29. pp. 3-20.

Guichon, N. (2003). "Compréhension de l'oral et apprentissage : L'exemple de Virtual Cabinet". Les Cahiers de l'APLIUT, vol. 23, no 1. pp. 67-76.

Hyland, K. (2005). Metadiscourse. London: Continuum Guides to Discourse.

Janeau, E. (2012). "Docteurs des écoles doctorales de l'Université Joseph Fourier 2004, 2005, 2006, 2007, 20083 ans après". Document interne.

Long, M. H. (2005). "Overview: A rationale for needs analysis and needs analysis research". Long, M. H. (dir.) Second Language Needs Analysis. Cambridge: Cambridge Applied Linguistics, pp. 1-18.

Narcy-Combes, M.-F. 2005. Précis de didactique : Devenir professeur de langues. Paris: Ellipses.

McNamara, T. (2000). Language testing. Oxford: Oxford University Press.

Ministère de l'Education Nationale (2012). "Certificats exigés à la titularisation des lauréats des concours du second degré". Consulté en décembre 2012 : http://www.education.gouv.fr/cid56637/certificats-exiges-a-latitularisation-des-laureats-des-concours-du-second-degre.html

Parlement Européen et le Conseil de l'Union Européenne. (2006). Recommandation du Parlement Européen et du Conseil du 18 décembre 2006 sur les compétences clés pour l'éducation et la formation tout au long de la vie. Consulté en avril 2012 : http://eur-lex.europa.eu/LexUriServ/LexUriServ.do? uri=OJ:L:2006:394:0010:0018:fr:PDF

Poteaux, N. (2003). "L'autoformation à l'université : De quelques éléments dialectiques". In Albero, B. (dir.). Autoformation et enseignement supérieur. Paris : Lavoisier.

Rademacher, W. \& Leitner, M. (2009). The Bologna Process in Higher Education in Europe: Key indicators on the social dimension and mobility. Consulté en avril 2012 : http:// www.ond.vlaanderen.be/hogeronderwijs/bologna/conference/ documents/2009_eurostat_eurostudent_social_dimension_and_mobility_indicators.pdf Räisänen, C., \& Fortanet-Gomez, I. (2008). "ESP teaching and learning in Western European higher education." In Räisänen, C. \& Fortanet-Gomez, I. (dirs). ESP in European higher education: Integrating language and content. Amsterdam: John Benjamins Publishing Company.

Shaw, P. (2011). "Conditions for success in lingua franca interaction". ASp : La revue de GERAS, $\mathrm{n}$ 60. pp. 65-79.

Swales, J. M. (2004). Genre Analysis: English in academic and research settings. Cambridge : Cambridge Applied Linguistics.

Taillefer, G. 2008. "Transformations, évolution : Un regard sur la dynamique de notre métier". Les Cahiers de l'APLIUT, vol. 27, n². pp. 49-65.

Taillefer, G. (2007). "The professional language needs of Economics graduates: Assessment and perspectives in the French context". English for Specific Purposes, n 26. pp. 135-155. 
Thematic Network project in the area of Languages - TNP3. (2008). Rapport de synthèse "Ouest" : Langues étrangères, facteur d'employabilité sur le marché du travail Européen. Consulté en décembre 2012 : http://www.tnp3-d.org/docs/tnp3d_sr_sp2_west_fr_0.pdf

Truchot, C. (1997). "The spread of English: From France to a more general perspective". World Englishes, vol. 16, n 1. pp. 65-76.

\section{ANNEXES}

Annexe 1 : Extrait règlement d'examen : CEVU du 29 juin 2006 et CA 11 juillet 2006

- "Le diplôme/grade de Master est délivré dès que, d'une part les conditions d'obtention du diplôme intermédiaire de maîtrise sont satisfaites et que d'autre part les échelons (semestres) 3 et $4 \mathrm{du}$ parcours ont été validés séparément"

- "L'échelon 4 ne peut être validé que si l'étudiant a fait la preuve dans son parcours de son aptitude à maîtriser au moins une langue étrangère (cette langue pouvant être le français pour les étudiants étrangers non francophones)". A compter de l'année universitaire 2008-2009, le niveau de qualification B2 du conseil de l'Europe dans les 3 champs de compétence définis par ALTE sera exigé.

- "Le jury de diplôme peut reconsidérer en fin de cursus l'ensemble du parcours de l'étudiant sur les quatre échelons (semestres) et décider de lui délivrer le diplôme et donc de lui reconnaître les 120 crédits de master, même si tous les échelons n'ont pas été acquis.

Lorsque l'étudiant n'a pas validé le niveau de qualification requis, le jury décide après avis d'une commission d'établissement siégeant pour l'ensemble des étudiants concernés dans les différentes mentions de master".

Annexe 2 : Questionnaire

Questionnaire Service des Langues Doctorat

1. Je possède :

$\square$ CLES2 ou $3 \square$ IELTS5+ $\square$ Toeic 750+ $\square$ TOEFL $\square$ Autre certificat/langue : Dans ma famille, nous parlons

2. Avec quelle fréquence utilisez-vous ces différentes compétences en anglais?

\begin{tabular}{|l|l|l|l|l|}
\hline & Tous les jours & Plusieurs fois/mois & Plusieurs fois/an & Rarement \\
\hline Lire & $\square$ & $\square$ & $\square$ & $\square$ \\
\hline Écouter & $\square$ & $\square$ & $\square$ & $\square$ \\
\hline Écrire & $\square$ & $\square$ & $\square$ & $\square$ \\
\hline Parler & $\square$ & $\square$ & $\square$ & $\square$ \\
\hline Lecture d'article scientifique & $\square$ & $\square$ & $\square$ & $\square$ \\
\hline Présentation orale & $\square$ & $\square$ & $\square$ & $\square$ \\
\hline
\end{tabular}




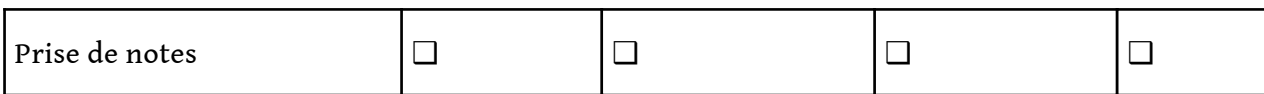

3. Avez-vous des difficultés pour utiliser ces différentes compétences en anglais?

\begin{tabular}{|l|l|l|l|}
\hline & Oui & Parfois & Non \\
\hline Lire & $\square$ & $\square$ & $\square$ \\
\hline Écouter & $\square$ & $\square$ & $\square$ \\
\hline Écrire & $\square$ & $\square$ & $\square$ \\
\hline Parler & $\square$ & $\square$ & $\square$ \\
\hline Lecture d'article scientifique & $\square$ & $\square$ & $\square$ \\
\hline Présentation orale & $\square$ & $\square$ & $\square$ \\
\hline Prise de notes & $\square$ & $\square$ & $\square$ \\
\hline
\end{tabular}

4. Actuellement, dans votre travail ou pour décrocher un travail, est-il nécessaire pour vous de progresser sur le(s) plan(s) des compétences en anglais de :

\begin{tabular}{|l|l|l|}
\hline Lire & Oui $\square$ & Non $\square$ \\
\hline Écouter & Oui $\square$ & Non $\square$ \\
\hline Écrire & Oui $\square$ & Non $\square$ \\
\hline Parler & Oui $\square$ & Non $\square$ \\
\hline Lecture d'article scientifique & Oui $\square$ & Non $\square$ \\
\hline Présentation orale & Oui $\square$ & Non $\square$ \\
\hline Prise de notes & Oui $\square$ & Non $\square$ \\
\hline
\end{tabular}

5. L'Université Joseph Fourier a fait le choix d'orienter l'enseignement de l'anglais en Master vers les objectifs suivants en anglais lié à la formation. Que pensez-vous de l'enseignement de ces objectifs?

- Anglais lié à votre formation

- Lecture d'article scientifique

- Présentation orale

- Prise de notes à partir de sources orales

Annexe 3 : Réponses des personnes liées au recrutement au sein des entreprises 


\section{Questionnaire DRH}

L'Université Joseph Fourier s'implique fortement dans l'enseignement des compétences en anglais à objectif professionnel. Le niveau d'anglais est testé en interne selon les critères reconnus au niveau européen. Si le niveau est validé, cela est noté sur le diplôme de Master (Bac+5). Les objectifs sont l'enseignement de l'anglais lié à la formation dont la lecture d'article scientifique, la présentation orale et la prise de notes à partir de sources orales. Nous faisons référence à ces objectifs sous l'appellation « Validation B2 ALTE Études ». Pour juger du bienfondé de la démarche et de l'atteinte de ces objectifs, nous souhaitons connaître votre avis. Nous évaluons le temps de remplir ce questionnaire à 10-15 minutes. Le Service des Langues vous remercie de votre participation au suivi professionnel des étudiant(e)s.

1. Dans la recherche d'un emploi arrive-t-il que le (la) candidate(e) ne soit pas embauché(e) à cause du niveau en anglais ... (cocher la case choisie)

\begin{tabular}{|l|l|l|}
\hline & Oui & Non \\
\hline en lecture & 7 & \\
\hline en compréhension auditive & 6 & \\
\hline en expression écrite & 7 & \\
\hline en expression orale & 6 & 2 \\
\hline en lecture d'articles scientifiques & 1 & 2 \\
\hline en présentation orale & 2 & $(1$ selon poste $)$ \\
\hline en prise de notes à partir de sources orales & & 3 \\
\hline
\end{tabular}

2. Quels items ont un effet positif sur l'embauche? (cocher la case choisie)

\begin{tabular}{|l|l|l|}
\hline & Oui & Non \\
\hline Bon niveau d'anglais & 7 & \\
\hline $\begin{array}{l}\text { Validation B2 ALTE Études à l'Université Joseph Fourier sur le diplôme de } \\
\text { Master. }\end{array}$ & 3 & \\
\hline Préparation au CLES2 ou 3 & 3 (dont un ? & \\
\hline IELTS5+ & ? & \\
\hline Toeic 750+ & 4 & \\
\hline
\end{tabular}


Autre certificat ou langue, quoi?

$\begin{array}{ll}2 & \\ 2 & \text { selon } \\ \text { poste }\end{array}$

3. Quels items ont un effet positif sur la performance professionnelle? (cocher la case choisie)

\begin{tabular}{|l|l|l|}
\hline & Oui & Non \\
\hline Bon niveau d'anglais & 7 & \\
\hline Préparation à la validation B2 ALTE Études à l'Université Joseph Fourier & & 1 \\
\hline Préparation au CLES2 ou 3 & & 1 \\
\hline Préparation à l'IELTS5+ & & 1 \\
\hline Préparation au Toeic 750+ & & 1 \\
\hline Préparation à un autre certificat ou langue, quoi ? & Selon le cas & 1 \\
\hline
\end{tabular}

4. Avec quelle fréquence utilise-t-on ces différentes compétences en anglais dans les professions scientifiques? (cocher la case choisie)

\begin{tabular}{|c|c|c|c|c|}
\hline & Tous les jours & Plusieurs fois/mois & Plusieurs fois/an & Rarement \\
\hline Lire & 6 & 1 & & \\
\hline Écouter & 4 & 2 & 1 & \\
\hline Écrire & 6 & 1 & & \\
\hline Parler & 4 & 2 & 1 & \\
\hline Lecture d'article scientifique & & $\begin{array}{l}4 \\
(1 \text { technique) }\end{array}$ & 1 & 2 \\
\hline Présentation orale & 1 & 3 & 2 & 1 \\
\hline Prise de notes & & & 1 & 1 \\
\hline
\end{tabular}

5. Quel pourcentage des candidats(es) titulaires d'un Master a des difficultés pour utiliser ces différentes compétences en anglais au niveau professionnel ? (cocher la case choisie)

\begin{tabular}{|l|l|l|l|}
\hline $0 \%-25 \%$ & $26 \%-50 \%$ & $51 \%-75 \%$ & $>75 \%$ \\
\hline
\end{tabular}




\begin{tabular}{|l|l|l|l|l|}
\hline Lire & 3 & & & \\
\hline Écouter & & 2 & 1 & \\
\hline Écrire & 1 & 2 & & \\
\hline Parler & & 3 & & \\
\hline Lecture d'article scientifique & 1 & 1 & & \\
\hline Présentation orale & & & 2 & \\
\hline Prise de notes & & & 2 & \\
\hline
\end{tabular}

6. Quelle importance accordez-vous, sur le plan professionnel, à l'utilisation de ces différentes compétences en langue étrangère ? (cocher la case choisie)

\begin{tabular}{|l|l|l|l|}
\hline & Importance élevée & Importance moyenne & Importance faible \\
\hline Lire & 7 & & \\
\hline Écouter & 5 & 2 & \\
\hline Écrire & 7 & & \\
\hline Parler & 5 & 2 & 1 \\
\hline Lecture d'article scientifique & 1 & 3 & 2 (dont 1 selon poste) \\
\hline Présentation orale & 2 & 3 & 4 \\
\hline Prise de notes & & & \\
\hline
\end{tabular}

\section{Anglais lié à votre formation (23 réponses)}

- Pas eu de formation.

- Je l'ai appris par l'intermédiaire de mes candidatures en post doc après la thèse. Je ne l'ai malheureusement jamais appris au cours d'un cursus universitaire.

- Inutile.

- L'enseignement de l'anglais en France est très mauvais, basé sur l'enseignement de la grammaire et des listes de vocabulaire que l'on n'utilisera jamais. Jamais on ne m'a enseigné les mots simples de tous les jours qui permettent d'initier une conversation. - Ça maintient un niveau basal en anglais et cela permet de ne pas décrocher totalement. Mais c'est bien tout ce que ça apporte. Le mieux est de partir à l'étranger.

- L'ensemble du cursus « anglais » que j'ai suivi au cours de mes études n'a jamais été efficace ni utile. J'ai vraiment appris l'anglais par moi-même en lisant des livres en anglais et surtout au cours de mon incursion en pays anglophone lors de mon Post doc. - L'anglais technique peut rapidement s'apprendre sur le tas. Il est plus utile d'avoir un anglais scientifique de base.

- Je pense que la compréhension et l'expression orale classiques sont beaucoup plus 
importantes qu'une formation en anglais liée au domaine de formation.

- Je pense qu'il faut insister sur l'enseignement de l'anglais, favoriser les stages à

l'étranger (M1 ou M2).

- Je n'ai pas suivi des formation en anglais dans le cadre de la thèse. Cependant les quatre points me semblent primordiaux dans le cadre de poursuite vers un emploi de maitre de conférences ou de chercheur.

- Bien (2).

- D'accord.

- OK.

- Oui!

- Enfin!

- Indispensable (3).

- Nécessaire.

- Très bien.

- Primordial (2).

Lecture d'articles scientifiques ( 24 réponses)

- Pas eu de formation.

- Inutile.

- Peu utile.

- On le fait déjà tous les jours dans le cadre de la thèse. Je ne pense pas que ce soit nécessaire d'avoir des cours.

- Article scientifique = anglais basique et formules récurrentes. Une formation est-elle vraiment indispensable?

- Moyen. J'étais suffisamment préparé pour lire l'anglais scientifique. Tenir une discussion courte était plus dur.

- Utile pour la recherche, mais pas pour l'obtention d'un travail hors de la recherche.

- La lecture d'articles scientifiques pose deux problèmes : celui de la langue et celui de l'aspect scientifique. Venant de classe préparatoire aux grandes écoles j'ai été un peu familiarisé avec la synthèse d'articles via les concours (essentiellement celui de l'ENS et celui des ENSI à l'époque). En ce qui concerne la langue, l'enseignement de l'anglais en France est toujours fait à partir d'articles d'actualité souvent politique et jamais d'articles scientifiques.

- Cette compétence est indispensable, mais son enseignement est-il nécessaire?

- On en a toujours besoin. Autant en faire pas mal pour vite arriver à ne plus avoir besoin de lire une publication avec un dictionnaire à côté !

- Utile.

- À développer.

- D'accord.

- OK.

- Oui.

- OK, déjà en place à mon époque.

- Bon enseignement.

- Nécessaire.

- Indispensable (5).

- Enfin!

Présentation orale (23 réponses) 
- Pas eu de formation.

- ma première présentation scientifique en anglais a été faite en-dehors du cadre de ma scolarité et je n'y avais jamais été préparée.

- Je l'ai appris sur le tas ayant des présentations et des conférences à faire. Les cours suivis lors de mon cursus universitaire n'ont jamais été utiles

- Ce doit être le rôle des encadrants de la thèse.

- En anglais je n'ai fait qu'une présentation quand j'étais en thèse et c'est moi-même qui me suis entraîné pour mon entretien d'embauche en Post doc. Un gros travail à faire par l'école doctorale sur ce point.

- Beaucoup de gens oublient qu'il est aussi important de savoir bien communiquer en anglais. C'est un bon exercice surtout si le sujet de communication est en rapport avec les cours ou les travaux de recherche.

- Utile.

- À développer.

- Bien.

- OK.

- D'accord.

- Très bien.

- Nécessaire.

- Indispensable (4).

- Passage obligé.

- Primordial.

- Enfin!

- Bonne idée pour progresser rapidement.

- Il faudrait soumettre les étudiants à cet exercice dès la Master !! !

- Très important. Seule la pratique permet d'acquérir une assurance parfaite.

Prise de notes à partir de sources orales (21 réponses)

- Pas eu de formation.

- Inutile.

- Aucune utilité.

- Je ne vois pas l'utilité.

- Peu pertinent à mon sens.

- Peu utile

- Moyenne aussi. Aucune formation à proprement parler pour la prise de notes, encore moins en anglais.

- La prise de notes est par nature permissive en ce qui concerne les fautes de langue. Je ne pense pas qu'il y ait un effort à faire dans cette direction. Le problème est plus la compréhension de la source orale que la reproduction sous forme de notes.

- Indispensable, mais découle du reste : si on parle couramment, cela ne pose pas plus de problème que le reste.

- Je trouve qu'on apprend beaucoup à suivre des cours en anglais.

- La prise de notes, on la fait comme on veut. Le plus important c'est de comprendre ce que disent les gens et de prendre les notes qui vous intéressent.

- Optionnel.

- D'accord.

- OK lors de conférences.

- OK. 
- Oui.

- Bonne initiative.

- À développer.

- Nécessaire.

- Indispensable.

- Enfin!

\section{RÉSUMÉS}

En 2002, la France a entériné l'objectif linguistique mis en place dans le cadre de la mobilité européenne en décrétant que le diplôme de master « ne peut être délivré qu'après validation de l'aptitude à maîtriser au moins une langue étrangère ». Depuis une décennie, très peu d'études ont examiné la manière d'évaluer cette aptitude ou les véritables besoins professionnels des diplômé-es, notamment dans les domaines des sciences. Dans ce cadre, l'Université Joseph Fourier a lancé une enquête auprès des docteurs en sciences trois ans après l'obtention du diplôme et auprès des responsables de ressources humaines parmi les établissements susceptibles de les recruter. L'objectif de la recherche est de cibler la corrélation entre les compétences de communication en anglais général ou scientifique et les besoins professionnels. Un t-test n'indique aucune différence significative $(\mathrm{p}=0,718)$ pour un sous-ensemble des 12 docteur-es possédant un certificat Toeic $(M=5,33 ; E T=1,83)$ et les 67 autres docteur-es $(M=5,11$; $\mathrm{ET}=2,02)$. C'est-à-dire que, en ce qui concerne la pratique et la maîtrise de l'anglais, il n'y a pas de différence significative entre les personnes possédant un Toeic (750+) et le reste de cette population. Les résultats montrent aussi qu'une bonne maîtrise de l'ensemble des compétences en anglais est importante. La lecture est un acte quotidien et pose peu de difficultés, l'expression orale est moins pratiquée et moins bien maitrisée et enfin les résultats concernant la prise de notes s'avèrent moins homogènes. Au-delà des compétences spécifiques, la maîtrise de l'anglais jouerait un rôle essentiel dans le parcours professionnel des docteur-es en sciences.

In 2002, France endorsed the language objective necessary to European mobility by publishing the decree that a Master's degree cannot be delivered until the capacity to master at least one foreign language has been validated. Over the last decade, few studies have examined the means to evaluate that aptitude or the actual professional needs of degree holders, especially within the sciences. Within this context, the University Joseph Fourier conducted a survey of doctors of science three years after graduation and the human resources personnel in companies likely to recruit this population. The aim of this study is to better understand the correlation between communication skills in general or scientific English and professional needs. A t-test showed no significant difference $(p=0.718)$ between the 12 persons having obtained a Toeic $(750+)$ certificate $(M=5.33 ; E T=1.83)$ and the 67 graduate students $(M=5.11 ; E T=2.02)$. In other words, there was no significant difference of ease or frequency of communicating in English between the persons having obtained a Toeic certificate and the rest of the population. The results also show that a good command of English is professionally important. Reading is a frequently used skill, which poses few problems, speaking is less frequent and shows weaker command and finally the results for note-taking are less homogeneous. Beyond the differences between skills, this data highlights the importance of mastering English for conferences or other international experiences as they appear to play an essential role in this public's careers. 
INDEX

Mots-clés : sciences, compétences, lingua franca, besoins professionnels

Keywords : Sciences, skills, lingua franca, professionnel language needs

\section{AUTEUR}

LAURA M. HARTWELL

Laura M. Hartwell est maître de conférence au laboratoire LIDILEM et enseigne à l'université Joseph Fourier à l'Université Grenoble Alpes.

Courriel : hartwell@ujf-grenoble.fr

Toile : http://w3.u-grenoble3.fr/lidilem/labo/web/presentation.php

Adresse : UJF Valence, Université Joseph Fourier, Site Briffaut, 38 rue Barthélemy de Laffemas, 26000 Valence cedex, France. 